ARTICLE

Received 29 Nov 2016 | Accepted 10 Mar 2017 | Published 25 May 2017

DOI: $10.1038 /$ ncomms15224

\title{
Negative Poisson's ratio in 1T-type crystalline two-dimensional transition metal dichalcogenides
}

Liping Yu${ }^{1}$, Qimin $\operatorname{Yan}^{1} \&$ Adrienn Ruzsinszky ${ }^{1}$

Materials with a negative Poisson's ratio, also known as auxetic materials, exhibit unusual and counterintuitive mechanical behaviour-becoming fatter in cross-section when stretched. Such behaviour is mostly attributed to some special re-entrant or hinged geometric structures regardless of the chemical composition and electronic structure of a material. Here, using first-principles calculations, we report a class of auxetic single-layer two-dimensional materials, namely, the 1T-type monolayer crystals of groups 6-7 transition-metal dichalcogenides, $\mathrm{MX}_{2}(\mathrm{M}=\mathrm{Mo}, \mathrm{W}, \mathrm{Tc}, \mathrm{Re} ; \mathrm{X}=\mathrm{S}, \mathrm{Se}, \mathrm{Te})$. These materials have a crystal structure distinct from all other known auxetic materials. They exhibit an intrinsic in-plane negative Poisson's ratio, which is dominated by electronic effects. We attribute the occurrence of such auxetic behaviour to the strong coupling between the chalcogen $p$ orbitals and the intermetal $t_{2 g}$-bonding orbitals within the basic triangular pyramid structure unit. The unusual auxetic behaviour in combination with other remarkable properties of monolayer two-dimensional materials could lead to novel multi-functionalities.

\footnotetext{
${ }^{1}$ Department of Physics, Temple University, Philadelphia, Pennsylvania 19122, USA. Correspondence and requests for materials should be addressed to L.Y. (email: yuliping@gmail.com).
} 
T he Poisson's ratio of a material characterizes its response to uniaxial load and is given by $v_{a b}=-\varepsilon_{b} / \varepsilon_{a}$, where $\varepsilon_{a}$ is an applied strain in the $a$ axis direction and $\varepsilon_{b}$ is the resulting strain in a transverse $b$ axis direction. Counter-intuitively, negative Poisson's ratio (auxetic) materials ${ }^{1}$ expand laterally when stretched and contract laterally when compressed. They can lead to enhanced mechanical properties, such as shear modulus ${ }^{2}$, indentation resistance ${ }^{3}$ and fracture toughness ${ }^{4}$. The unusual auxetic effect itself and concomitant enhancements in other material properties offers enormous potential in many technologically important applications ${ }^{5-7}$, such as biomedicine ${ }^{8}$, sensors $^{9}$, fasteners ${ }^{10}$ and protective equipments ${ }^{11}$.

Auxetic effect has been reported in a number of natural and man-made materials and structures in bulk form ${ }^{5,6,12,13}$, for example, cubic metals ${ }^{14,15}, \alpha$-cristobalite $\left(\mathrm{SiO}_{2}\right)^{16}, \alpha$-TeO ${ }_{2}$ (ref. 17), the zeolite mineral natrolite ${ }^{18}$, honeycombs ${ }^{19}$, foams ${ }^{7}$, microporous polymers ${ }^{20,21}$, composites ${ }^{22,23}$, ceramics $^{24}$, molecular auxtics ${ }^{25}$, metal-organic frameworks ${ }^{26}$, bucklicrystals ${ }^{27}$ and origami structures ${ }^{28-30}$. Geometric considerations dominate the literature in understanding such auxetic effects and designing new auxetic materials. For most of these auxetic materials, the auxetic effect is explained by some special re-entrant structure or the crystal structure that can be viewed as being made up of rigid building blocks linked by flexible hinges ${ }^{1,19,31-33}$, independent of their chemical composition and electronic structure.

Auxetic effect has also been recently reported in several monolayer two-dimensional (2D) materials. For example, the out-of-plane negative Poisson's ratio was discovered in phosphorene $\mathrm{e}^{34,35}, \mathrm{GeS}^{36}$ and monolayer arsenic ${ }^{37}$. The in-plane negative Poisson's ratio was also predicted in borophene ${ }^{38}$ and three theoretically proposed but not-yet-synthesized materials (that is, the penta-graphene ${ }^{39,40}, h \alpha$-silica ${ }^{41}$ and $\mathrm{Be}_{5} \mathrm{C}_{2}$ (ref. 42)). Similar to that in the bulk auxetic materials, the auxetic behaviour in these $2 \mathrm{D}$ materials is also considered to originate mainly from the puckered or buckled crystal structure.

In this study, using quantum mechanical first-principles calculations (see Methods section), we report a class of auxetic single-layer 2D materials with an intrinsic in-plane negative Poisson's ratio. They differ from other known auxetic materials not only in their crystal structure but also in the microscopic origin of auxetic behaviour. These materials are the 1T-type crystalline monolayers of groups 6-7 transition metal dichalcogenides, $1 \mathrm{~T}-\mathrm{MX}_{2}(\mathrm{M}=\mathrm{Mo}, \mathrm{W}, \mathrm{Tc}, \mathrm{Re} ; \mathrm{X}=\mathrm{S}, \mathrm{Se}, \mathrm{Te})$. In contrast to those known bulk or $2 \mathrm{D}$ auxetic materials, the in-plane auxetic behaviour discovered in groups 6-7 1T- $\mathrm{MX}_{2}$ cannot be explained merely from their geometric structure because the non-auxetic behaviour is also found in other groups of $\mathrm{MX}_{2}$ compounds with the same 1T-type structure. This dichotomy between auxetic and non-auxetic behaviour in the $1 \mathrm{~T}-\mathrm{MX}_{2}$ compounds is explained by their distinct electron structures. The in-plane stiffness of those $1 \mathrm{~T}-\mathrm{MX}_{2}$ materials is predicted to be order of $10^{2} \mathrm{GPa}$, at least three orders of magnitude higher than man-made auxetic materials. The high in-plane stiffness and the auxetic behaviour in combination with other remarkable electronic and optoelectronic properties of the single-layer $2 \mathrm{D}$ materials ${ }^{43}$ could lead to novel multifunctionalities, such as nanoscale auxetic electrodes and sensors.

\section{Results}

Crystal structure. The single layers of 2D transition metal dichalcogenides are formed by a hexagonally packed layer of metal (M) atoms sandwiched between two layers of chalcogen (X) atoms (Fig. 1). Each chalcogen atom forms the apex of a triangular pyramid that has three metal atoms at its base. The symmetry of the chalcogen array about each metal atom is either octahedral or trigonal prismatic. The former is often referred to as the $1 \mathrm{~T}$ phase, whereas the latter as the $1 \mathrm{H}$ phase. Depending on the combination of the metal and chalcogen elements, one of the two phases is thermodynamically preferred. Most group- $6 \mathrm{MX}_{2}$ compounds thermodynamically prefer the $1 \mathrm{H}$ phase ${ }^{44}$, but the metastable $1 \mathrm{~T}$ phase is also observed ${ }^{45-47}$. For other groups of layered $\mathrm{MX}_{2}$ compounds, most crystallize in the high-symmetry $1 \mathrm{~T}$ or low-symmetry distorted $1 \mathrm{~T}$ phase ${ }^{44,48}$. The $1 \mathrm{H}-\mathrm{MX}_{2}$ compounds are known to be non-auxetic in the plane due to their hexagonal in-plane crystalline structure. We hence focus on 42 monolayer $\mathrm{MX}_{2}$ compounds in the high-symmetry $1 \mathrm{~T}$ phase (Table 1).

Poisson's ratio results. Figure 2a shows our calculated Poisson's ratio results $\left(v_{a b}\right)$ for 42 1T- $\mathrm{MX}_{2}$ compounds in the $b$ axis direction subjected to a 5\% tensile strain applied along the $a$ axis direction. Remarkably, we find that the sign of Poisson's ratio strongly depends on the $d$-electron count. All 12 1T-MX compounds from group $6\left(d^{2}\right)$ and group $7\left(d^{3}\right)$ exhibit negative Poisson's ratios, ranging from -0.03 to -0.37 . Seven of them (that is, $\mathrm{TcTe}_{2}, \mathrm{ReTe}_{2}, \mathrm{WTe}_{2}, \mathrm{WSe}_{2}, \mathrm{MoSe}_{2}, \mathrm{ReS}_{2}$ and $\mathrm{TcS}_{2}$ ) have a Poisson's ratio $<-0.1$, higher in magnitude than that of borophene $(-0.04 \text { along } a \text { and }-0.02 \text { along } b)^{38}$, rendering them more promising candidates for specific applications in mechanical nanodevices. For other groups of $1 \mathrm{~T}-\mathrm{MX}_{2}$ compounds, we find positive Poisson's ratios ranging from 0.09 to 0.53 .

Figure $2 \mathrm{~b}, \mathrm{c}$ shows our calculated Poisson's ratios $\left(v_{a b}\right.$ and $\left.v_{b a}\right)$ as a function of applied strain in two example compounds, non-auxetic $\mathrm{ZrS}_{2}$ and auxetic $\mathrm{MoS}_{2}$. For both compounds, Poisson's ratio varies slowly as applied strain goes from $-5 \%$ to $5 \%$, suggesting a dominant linear elastic behaviour within the strain range considered. (Note the Poisson's ratio at a large strain (that is, $>5 \%$ or $<-5 \%$ ) may strongly depend on the strain. This behaviour is not pursued in this work since such large strains are often experimentally inaccessible.) The small differences between $v_{a b}$ and $v_{b a}$ reflect a nearly isotropic auxetic or non-auxetic behaviour inside the 1T-structure plane. Therefore, the $d$-electron count dependence of the sign of Poisson's ratio as shown in Fig. 2a does not change with respect to the amount of the applied strain within the linear elastic range and the loading direction inside the plane.

Stiffness. To compare the stiffness (Young's modulus) of a singlelayer material with bulk materials, we calculate its $3 \mathrm{D}$ in-plane stiffness $\left(Y_{3 \mathrm{D}}\right)$ from 2D in-plane stiffness $\left(Y_{2 \mathrm{D}}\right)$ and effective layer thickness $(t)$ via $Y_{3 \mathrm{D}}=Y_{2 \mathrm{D}} / t$. The $Y_{2 \mathrm{D}}$ is directly derived from first-principles total energies as a function of uniaxial strain. The effective layer thickness $t$ can also be uniquely determined from first-principles calculated bending energy ${ }^{49}$. Here for simplicity, we approximate $t$ as $t=t_{0}+0.8 \AA$, where $t_{0}$ is the distance between the top and bottom chalcogen atom layers and the $0.8 \AA$ is the total effective decay length ( $0.4 \AA$ in each layer side) of electron density into the vacuum. The $0.8 \AA$ is derived from the first-principles calculated layer thickness for $1 \mathrm{H}-\mathrm{MoS}_{2}$ (ref. 49). $\mathrm{MSe}_{2}$ and $\mathrm{MTe}_{2}$ may have different decay lengths than $\mathrm{MS}_{2}$. However, such difference should be less than one time of magnitude. Hence, using a different decay length does not induce one time of magnitude difference in the calculated 3D in-plane stiffness.

Table 1 shows that the 3D in-plane stiffness of almost all $1 \mathrm{~T}-\mathrm{MX}_{2}$ compounds lies in between 100 and $300 \mathrm{GPa}$. Among the auxetic $d^{2}-d^{3} 1 \mathrm{~T}-\mathrm{MX}_{2}$ compounds, $\mathrm{WS}_{2}$ and $\mathrm{ReSe}_{2}$ are the stiffest, having a stiffness of $\sim 290 \mathrm{GPa}$; $\mathrm{TcTe}_{2}$ is the softest, having a stiffness of $\sim 80 \mathrm{GPa}$. Man-made auxetic materials typically have a stiffness in the range from $\sim 10^{-5}$ to $\sim 1 \mathrm{GPa}$, and naturally occurring auxetic bulk solids exhibit a stiffness of 
a



b

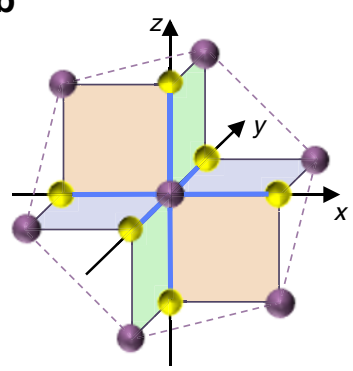

C

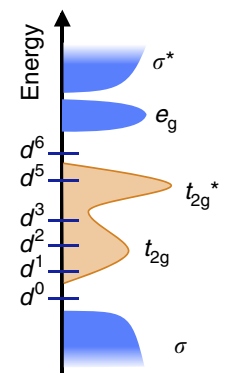

d

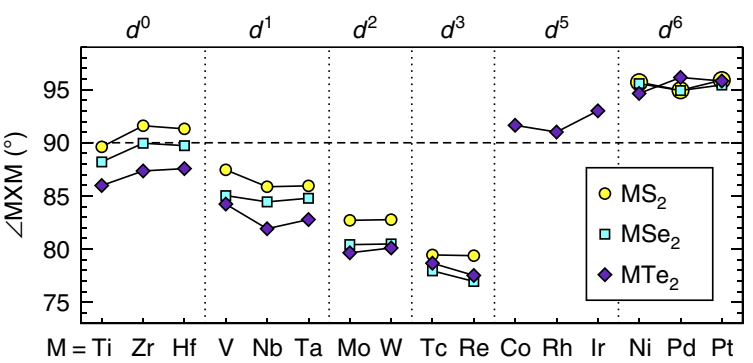

Figure 1 | Structure of monolayer $\mathbf{1 T -} \mathbf{M X}_{\mathbf{2}}$. (a) Crystal structure. The basic $X-M_{1}-M_{2}-M_{3}$ triangular pyramid unit is marked. The rectangular outline displays the unit cell adopted in our calculation. It contains two $\mathrm{MX}_{2}$ formula units. (b) Local structure of $\mathrm{M}$-centred octahedron. The metal atoms form three one-dimensional chains in the directions of $y=x, z=y$ and $z=-x$ in the local reference frame. The $M-M$ interaction is through the $t_{2 g}$-orbital coupling. (c) Schematic configuration of DOS showing the gradual filling of $d$ orbitals from group $4\left(d^{0}\right)$ to group $10\left(d^{6}\right) 1 \mathrm{~T}-\mathrm{MX}_{2}$. The horizontal bars denote the corresponding Femi level of the system. $t_{2 g}$ and $t_{2 g}^{\star}$ correspond to the intermetal $t_{2 g}$-bonding and $t_{2 g}$-antibonding states, respectively.

(d) Predicted $\mathrm{M}-\mathrm{X}-\mathrm{M}$ bond angles in the relaxed structure of strain-free $1 \mathrm{~T} M \mathrm{X}_{2}$. Note, in the triangular pyramid as shown in Fig. 1a,

$\angle M_{1} X M_{2}=\angle M_{2} X M_{3}=\angle M_{3} X M_{1}=\angle M X M$.

Table 1 | Predicted in-plane stiffness for 42 monolayer $1 \mathrm{~T}-\mathrm{MX}_{2}$ compounds.



The 3D in-plane stiffness (Young's Modulus) $\left(Y_{3 D}\right)$ is calculated from 2D in-plane stiffness $\left(Y_{2 D}\right)$ divided by the effective layer thickness $t$ given by $t=t_{0}+0.8 \AA$, where $t_{0}$ is the distance between the top and bottom chalcogen atom layers.

$\sim 10^{1}-10^{2} \mathrm{~Pa}$ (ref. 50). Therefore, even considering the uncertainty of our calculated $3 \mathrm{D}$ stiffness (less than one order of magnitude) that may be caused by using different approximations for effective layer thickness, the 3D stiffness values predicted for $1 \mathrm{~T}-\mathrm{MX}_{2}$ compounds are among the highest in the naturally occurring crystalline solids and are at least three orders of magnitude higher than man-made auxetic materials.

The fact that both auxetic and non-auxetic materials are found in the same 1T-structure type implies that the auxetic effect is not a purely geometric property. The $d$-electron count dependence of electronic structure must be involved. In the $1 \mathrm{~T}$ structure, the $d$ orbitals of the octahedrally coordinated transition metal split into two groups, $d_{x y, y z, z x}\left(t_{2 \mathrm{~g}}\right)$ and $d_{x^{2}-y^{2}, z^{2}}\left(e_{\mathrm{g}}\right)$. In what follows, we shall show that (i) transition metals interact with each other through $t_{2 \mathrm{~g}}$-orbital coupling, and (ii) the coupled $t_{2 \mathrm{~g}}$ orbitals are further coupled with the lone-pair electrons of chalcogen atoms. It is the gradual filling of such $t_{2 \mathrm{~g}}-p$-hybridized bands that leads to the different behaviour of Poisson's ratio. 

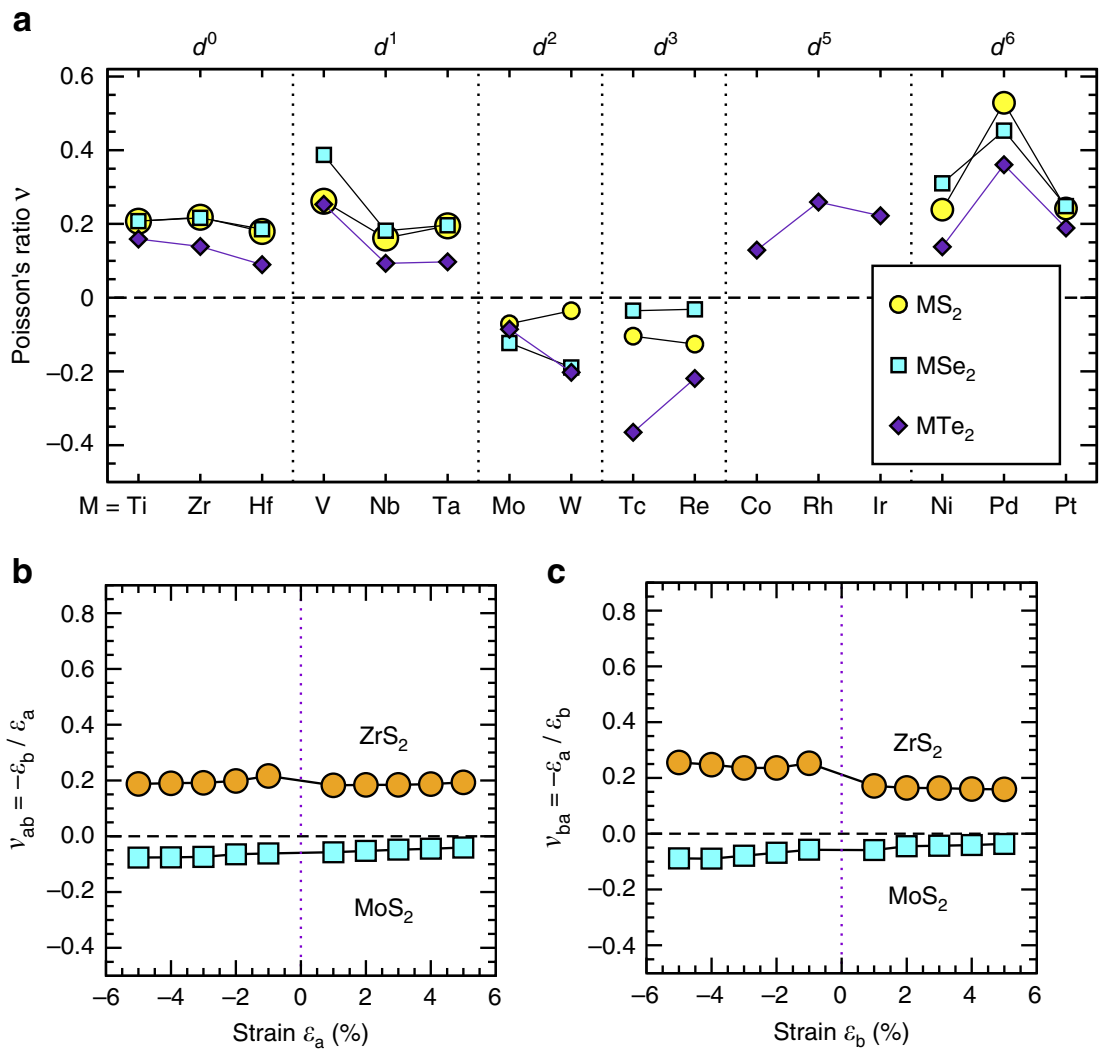

Figure 2 | Poisson's ratios. (a) Poisson's ratio, $v_{a b}=-\varepsilon_{b} / \varepsilon_{a}$, calculated for a $5 \%$ strain applied along the $a$ axis (that is, $\varepsilon_{a}=5 \%$ ). (b,c) Poisson's ratios for $\mathrm{ZrS}_{2}$ and $\mathrm{MoS}_{2}$ as a function of strain applied along the $a$ axis (b) and $b$ axis (c).

Intermetal $t_{2 \mathrm{~g}}$-orbital coupling. In the ideal $1 \mathrm{~T}$ phase, the M-centred octahedra share edges, forming three one-dimensional M-chains along the directions of lines $y=x, y=z$, and $z=-x$, respectively, within the local reference frame of the octahedra (Fig. 1b). The metal atoms can interact with each other through the coupling between their $t_{2 \mathrm{~g}}$ orbitals. This coupling gives rise to $t_{2 \mathrm{~g}}$-bonding states and $t_{2 \mathrm{~g}}$-antibonding states, with no energy gap in between due to the weak coupling nature. The $t_{2 \mathrm{~g}}$ states are mostly located within the gap between the bonding and antibonding bands of the $\mathrm{M}-\mathrm{X}$ bonds (Fig. 1c).

The progressive filling of these $t_{2 \mathrm{~g}}$ bands from group $4\left(d^{0}\right)$ to group $10\left(d^{6}\right)$ species leads to different $\mathrm{M}-\mathrm{M}$ bonding or antibonding character at the Fermi level. In $d^{1}-d^{3} 1 \mathrm{~T}-\mathrm{MX}_{2}$, the Fermi level crosses the $t_{2 \mathrm{~g}}$-bonding states; the highest occupied bands close to the Fermi-level thus exhibit a stronger bonding character as we go from $d^{1}$ to $d^{3}$. This bonding character attracts the metal atoms towards each other, leading to an intermetal distance shorter than that in the ideal $1 \mathrm{~T}$ structure. In $d^{5}-d^{6}$ $1 \mathrm{~T}-\mathrm{MX}_{2}$, since the $t_{2 \mathrm{~g}}$ bonding states can accommodate up to six electrons (three from each metal), all $t_{2 \mathrm{~g}}$-bonding states are filled and the Fermi level crosses the $t_{2 \mathrm{~g}}$-antibonding states. Hence the highest occupied bands in the vicinity of the Fermi level exhibit antibonding character, repelling metal atoms from each other.

The existence of the intermetal $t_{2 \mathrm{~g}}$-orbital interactions is reflected by the $d$-electron count dependence of the $\mathrm{M}-\mathrm{X}-\mathrm{M}$ bond angles $(\angle M X M)$ as illustrated in Fig. 1d. The ideal $1 T$ phase has regular octahedra with $\angle M X M=90^{\circ}$. In the $d^{0}$ $1 \mathrm{~T}-\mathrm{MX}_{2}$ compounds, the $\angle \mathrm{MXM}$ deviates least from $90^{\circ}$. This is expected since all $t_{2 \mathrm{~g}}$ states are almost completely unoccupied and the intermetal $d-d$ interaction is marginal. For the $d^{1}-d^{3}$ $1 \mathrm{~T}-\mathrm{MX}_{2}$, all have acute $\angle \mathrm{MXM}$, decreasing with the increasing $d$-electron count. This trend arises from the increasing intermetal $t_{2 \mathrm{~g}}$-bonding character in going from $d^{1}$ to $d^{3}$, which shortens the intermetal distance. In the $d^{5}-d^{6} 1 \mathrm{~T}-\mathrm{MX}_{2}$, the $\angle \mathrm{MXM}$ jumps up to over $90^{\circ}$, consistent with the intermetal $t_{2 \mathrm{~g}}$-antibonding character.

Figure 1d also shows that the chalcogen atoms have minor effect on $\angle M X M$ compared with the transition metals with different $d$-electron counts, but a trend can still be observed: the $\angle M X M$ decreases with increasing atomic number of the chalcogen. For example, the $\angle \mathrm{MXM}$ of $\mathrm{TiS}_{2}, \mathrm{TiSe}_{2}$ and $\mathrm{TiTe}_{2}$ decreases from $89.6^{\circ}$ to $88.2^{\circ}$ to $86.0^{\circ}$. This trend is not associated with the intermetal $t_{2 \mathrm{~g}}$-orbital interaction; instead it is intrinsic to the spatial distribution of the lone-pair charge density relative to that of the M-X bonds around the chalcogen.

$\boldsymbol{t}_{2 \mathrm{~g}}-\boldsymbol{p}$-orbital coupling. The intermetal $t_{2 \mathrm{~g}}$ orbitals are further coupled with chalcogen $p$ orbitals in $1 \mathrm{~T}-\mathrm{MX}_{2}$. It can be seen from their projected density of states (DOS) as shown in Fig. 3. In the $4 d$ transition metal disulfides with the ideal $1 \mathrm{~T}$ structure, we find that the DOS of sulfur $3 p$ and metal $t_{2 \mathrm{~g}}$ states overlap, as manifested by their similar DOS peak shapes and positions in energy. The $t_{2 \mathrm{~g}}-p$-orbital overlap is marginal in $d^{0} \mathrm{ZrS}_{2}$, but it increases quickly in going from $d^{\mathrm{t}} \mathrm{NbS}_{2}$ to $d^{6} \mathrm{PdS}_{2}$. This trend is clear not only in the energy range from -12 to $-7 \mathrm{eV}$, where the major peaks of $3 p$ DOS are located, but also near the Fermi level.

The $t_{2 \mathrm{~g}}-p$-orbital interaction is attractive because the $\mathrm{X}$ ligand has one lone electron pair and acts as a sigma donor. In $d^{1}-d^{3}$ $\mathrm{MX}_{2}$, the $t_{2 \mathrm{~g}}-p$ coupling force draws atom $\mathrm{X}$ towards the intermetal bond centres, because the $t_{2 \mathrm{~g}}$ states are the intermetal bonding states spreading over the M-M bond centers. In $d^{5}-d^{6}$ $\mathrm{MX}_{2}$, the $t_{2 \mathrm{~g}}-p$ coupling force attracts atoms $\mathrm{M}$ and $\mathrm{X}$ towards each other, because the $t_{2 \mathrm{~g}}$ states are antibonding and localized near the metal atoms. The $d$-electron count dependence of $t_{2 \mathrm{~g}}-p$ interaction direction plays a key role in determining the structure deformation presented below. 


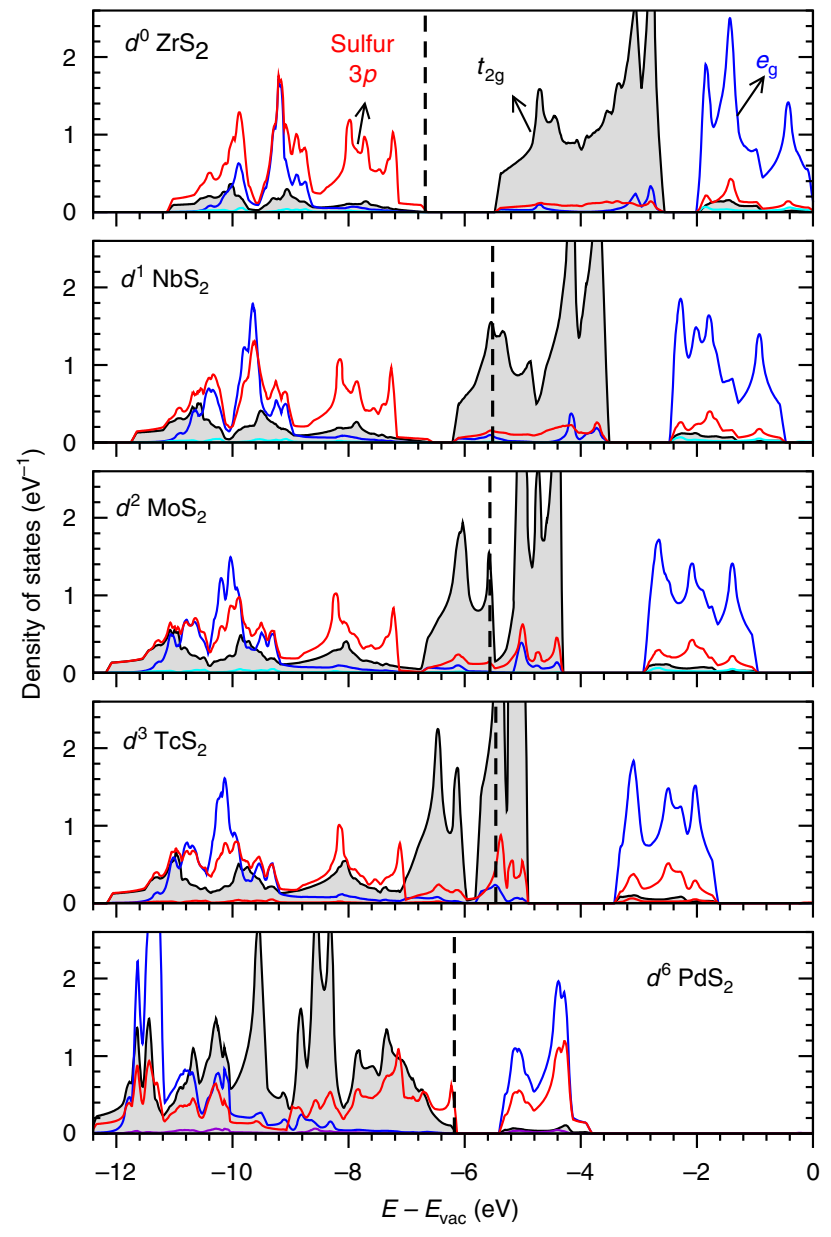

Figure 3 | DOS of $\mathbf{4 d} \mathbf{M S}$ in the ideal 1T structure. The $t_{2 g}-p$ orbital coupling manifests itself in the overlap of their DOS. The local reference frame in the octahedral is used for projecting DOS. The DOS shown in the figure are $t_{2 g}=d_{x y}+d_{y z}+d_{z x}, e_{g}=d_{x^{2}-y^{2}}+d_{z^{2}}$ and $p=p_{x}+p_{y}+p_{z}$. The vertical dashed lines show the position of Fermi level. The energy is aligned to the vacuum level.

Deformation mechanism. To understand the microscopic origin of Poisson's ratios, let us now look into the resulting structural relaxation subjected to a tensile strain applied along the $a$ axis. Due to the centrosymmetric nature of the $1 \mathrm{~T}$ phase, the whole relaxation process manifests itself in the triangular pyramid unit as illustrated in Fig. 4. For the stretch along the $M_{1}-M_{3}$ axis (that is, axis $a$ ), the resulting relaxation involves only atoms $\mathrm{M}_{2}$ and $\mathrm{X}$ moving inside the $\mathrm{Q}-\mathrm{X}-\mathrm{M}_{2}$ plane. Hence, two relations always hold during relaxation: $d_{\mathrm{M}_{1} \mathrm{M}_{2}}=d_{\mathrm{M}_{2} \mathrm{M}_{3}}$ and $\angle \mathrm{M}_{1} \mathrm{XM}_{2}=\angle \mathrm{M}_{3} \mathrm{XM}_{2}$.

We analyse the relaxation process by decomposing it into three consecutive steps: (i) atom $\mathrm{X}$ relaxes along the line $\mathrm{Q}-\mathrm{X}$, (ii) atom $X$ rotates around the $M_{1}-M_{3}$ axis, and (iii) atom $M_{2}$ relaxes along the line $\mathrm{Q}-\mathrm{M}_{2}$. In the first two steps, the lattice constant $b$ is fixed to the value found in the relaxed strain-free $1 \mathrm{~T}$ structure. In the third step, $b$ varies as atom $\mathrm{M}_{2}$ moves along the $\mathrm{Q}-\mathrm{M}_{2}$ line, leading to different Poisson's ratio behaviour.

Figure 4 shows the detailed structural relaxation in the three consecutive steps described above for $1 \mathrm{~T}-\mathrm{MX}_{2}$ with $\angle \mathrm{QXM}_{2}<90^{\circ}$ (Fig. 4a) and with $\angle \mathrm{QXM}_{2}>90^{\circ}$ (Fig. 4b) separately. Each step can be understood in the way that atom $\mathrm{X}$ (or atoms $\mathrm{X}$ and $\mathrm{M}_{2}$ ) relaxes to conserve the $\mathrm{M}-\mathrm{X}$ bond length $\left(d_{\mathrm{MX}}\right)$ since $d_{\mathrm{MX}}$ is energetically dominant. After the first two steps of the relaxation, it can be seen that (i) both $d_{\mathrm{M}_{1} \mathrm{M}_{2}}$ (also
$d_{\mathrm{M}_{2} \mathrm{M}_{3}}$ ) and $\angle \mathrm{M}_{1} \mathrm{XM}_{2}$ and $\angle \mathrm{M}_{3} \mathrm{XM}_{2}$ (Supplementary Fig. 1) increase in all $1 \mathrm{~T}-\mathrm{MX}_{2}$ compounds no matter whether $\angle \mathrm{QXM}_{2}$ is larger or smaller than $90^{\circ}$, and (ii) $\angle X Q M_{2}$ increases in the $1 \mathrm{~T}-\mathrm{MX}_{2}$ with $\angle \mathrm{QXM}_{2}<90^{\circ}$ but decreases in the $1 \mathrm{~T}-\mathrm{MX}_{2}$ with $\angle \mathrm{QXM}_{2}>90^{\circ}$ (Supplementary Fig. 1). The changes in $d_{\mathrm{M}_{1} \mathrm{M}_{2}}$ and $d_{\mathrm{M}_{2} \mathrm{M}_{3}}, \angle \mathrm{M}_{1} \mathrm{XM}_{2}$ and $\angle \mathrm{M}_{2} \mathrm{XM}_{3}$ and $\angle \mathrm{XQM}$ thus store the strain energy, which will be partially released in the subsequent third step relaxation.

The third-step relaxation determines the sign of Poisson's ratio. The negative Poisson's ratio of $d^{2}-d^{3} \mathrm{MX}_{2}$ can be attributed to the strong $t_{2 \mathrm{~g}}-p$-orbital coupling. Such strong coupling implies a large amount of strain energy stored in the decreased $\angle X \mathrm{XM}_{2}$ after the second step. This part of strain energy will be released in this third step through atom $\mathrm{M}_{2}$ relaxing along the increased $b$-lattice direction, leading to a negative Poisson's ratio. The strength of $t_{2 \mathrm{~g}}-p$-orbital coupling depends not only on the $d$-electron count of the transition metal but also on the chalcogen atom. This dependence explains why the Poisson's ratio of the compounds from same $d^{2}$ or $d^{3}$ group also differs from one another as shown in Fig. 2a.

For $d^{0}-d^{1} \mathrm{MX}_{2}$, the positive Poisson's ratio results from the marginal or weak intermetal $t_{2 \mathrm{~g}}$ coupling and $t_{2 \mathrm{~g}}-p$ coupling. Such weak couplings imply that the strain energy stored in $d_{\mathrm{M}_{1} \mathrm{M}_{2}}$ and $d_{\mathrm{M}_{2} \mathrm{M}_{3}}$ and $\angle \mathrm{XQM} \mathrm{M}_{2}$ is also marginal or small. The major strain energy that can be released in the third step is thus stored in the increased $\angle M_{1} X_{2}$ and $\angle M_{2} X M_{3}$. Therefore, it is energetically favourable that atom $\mathrm{M}_{2}$ relaxes in the $b$-decreasing direction, reducing the increase in $\angle M_{1} X_{2}$ and $\angle M_{2} X_{3}$, and resulting in a positive Poisson's ratio. For $d^{5}-d^{6} \mathrm{MX}_{2}$, the positive Poisson's ratio originates from the fact that the $t_{2 \mathrm{~g}}-p$ coupling aligns along the $\mathrm{M}-\mathrm{X}$ bond and does not energetically affect the change in $\angle X \mathrm{XM}_{2}$. In other words, the strain energy stored in the decreased $\angle X Q M_{2}$ is small. Since the $t_{2 \mathrm{~g}}$ antibonding is also generally weak, the relaxation of atom $\mathrm{M}_{2}$ is energetically favourable in the $b$-decreasing direction, giving rise to a positive Poisson's ratio. This deformation mechanism is similar to that in $d^{0}-d^{1}$ compounds.

Simply saying, the negative Poisson's ratio in $d^{2}-d^{3} \mathrm{MX}_{2}$ results from the strong attractive coupling between the intermetal $t_{2 \mathrm{~g}}$-bonding states and the $\mathrm{X} p$ states, which prevents atoms $\mathrm{X}$ and $M_{2}$ relaxing toward the $\angle X \mathrm{XM}_{2}$-increasing direction. The positive Poisson's ratio arises from lack of such strong $t_{2 \mathrm{~g}}-p$ coupling in other groups of $1 \mathrm{~T}-\mathrm{MX}_{2}$.

\section{Discussion}

The monolayer $\mathrm{MX}_{2}$ materials involve transition metals where strong correlation effects may be not well captured by the new strongly constrained and appropriately normed (SCAN) metaGGA functional. To check the robustness of our results, we also calculated the Poisson's ratio for $12 d^{2}-d^{3} \mathrm{MX}_{2}$ by using the HSE06 hybrid functional ${ }^{51}$. The results are summarized in Supplementary Table 1. It shows that the Poisson's ratio of eight 1T-MX $\mathrm{MX}_{2}$ compounds (that is, $\mathrm{MoSe}_{2}, \mathrm{MoTe}_{2}, \mathrm{WSe}{ }_{2}, \mathrm{WTe}_{2}$, $\mathrm{TcTe}_{2}, \mathrm{ReS}_{2}, \mathrm{ReSe}_{2}, \mathrm{ReTe}_{2}$ ) remains negative, whereas for other four compounds (that is, $\mathrm{MoS}_{2}, \mathrm{WS}_{2}, \mathrm{TcS}_{2}, \mathrm{TcSe}_{2}$ ) their Poisson's ratio changes the sign from negative to slightly positive, which is still very interesting and useful for applications. Although it is found that the SCAN lattice constants agree better with experiment than the HSE06 ones for most of the compounds listed in this table, it is uncertain whether SCAN predicts a more accurate Poisson's ratio than HSE06 since the semilocal SCAN functional could make larger density-driven error in the energy than HSE06 does for the system under stretching ${ }^{52}$. This uncertainty calls for experimental validation and further theoretical study. Nevertheless, the auxetic behaviour we find is 
a

Atom $\mathrm{M} 2$ relaxes along the line $\mathrm{Q}-\mathrm{M}_{2}$.

Atom $X$ relaxes along the line $Q-X$

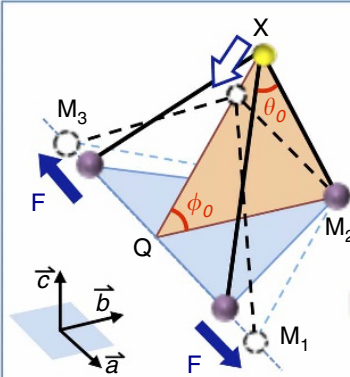

In this step, both $\angle X Q M_{2}$ and $d_{\mathrm{M}_{2} \mathrm{Q}}$ (that is, $b / 2$ ) are fixed.

- Before stretch and relaxation: $d_{\mathrm{M}_{1} \mathrm{X}}=d_{\mathrm{M}_{2} \mathrm{X}}=d_{\mathrm{M}_{3} \mathrm{X}}=d_{0}$ $\angle \mathrm{M}_{1} \mathrm{XM}_{2}=\angle \mathrm{M}_{3} \mathrm{XM}_{2}=\angle \mathrm{M}_{1} \mathrm{XM}_{2}=\theta_{0}$ $\angle \mathrm{XQM}_{2}=\phi$

- After this step: $d_{\mathrm{M}_{2} \mathrm{X}}<d_{0}$ because $\angle \mathrm{QXM} \mathrm{M}_{2}<90^{\circ}$.
Atom $X$ rotates around the $M_{1}-M_{3}$ axis
In this step, atom $X$ also rotates to conserve the $M-X$ bond length as atom $\mathrm{M}_{2}$ moves.

- Negative Poisson's ratio $\left(d^{2}-d^{3} \mathrm{MX}_{2}\right)$

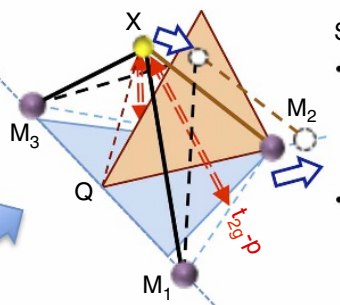

Strong $t_{2 g}-p$ coupling:

- reduces $\angle X Q_{2}$, releasing the strain energy stored in the increased $\angle \mathrm{XQM}_{2}$

- favours atom $M_{2}$ to relax in the $b$ increasing direction

- Positive Poisson's ratio $\left(d^{1} \mathrm{MX}_{2}\right)$

In this step, both $d_{\mathrm{QX}}$ and $d_{\mathrm{M}_{2} \mathrm{Q}}$ are fixed. After this step:

- $\angle \mathrm{XQM}_{2}>\phi_{0}$, as a result of the relaxation of the shortened $\mathrm{M}_{2}$ $\mathrm{X}$ bond $\left(d_{\mathrm{M}_{2} \mathrm{X}}<d_{0}\right)$.

- $\angle \mathrm{M}_{1} \mathrm{XM}_{2}=\angle \mathrm{M}_{3} \mathrm{XM}_{2}>\theta_{0}$ because the stretch increases $d_{\mathrm{M}_{1} \mathrm{M}_{2}}$ and $d_{\mathrm{M}_{2} \mathrm{M}_{3}}$ but $d_{\mathrm{MX}}$ remains close to $d_{0}$ after relaxation.

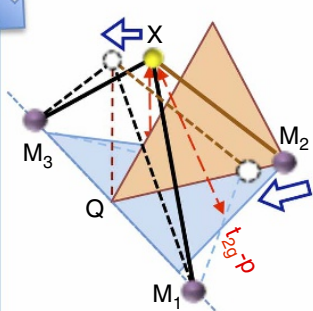

Weak $t_{2 \mathrm{~g}}-p$ coupling:

- implies that little strain energy can be stored in the change of $\angle X Q M_{2}$

- favours $M_{2}$ to relax in the $b$-decreasing direction, reducing $\angle \mathrm{M}_{1} \mathrm{XM}_{2}$ and $\angle \mathrm{M}_{3} \mathrm{XM}_{2}$ and releasing the strain energy stored in the increased $\angle \mathrm{M}_{1} \mathrm{XM}_{2}$ and $\angle \mathrm{M}_{3} \mathrm{XM}_{2}$.

b

\section{$\angle \mathrm{QXM}_{2}<90^{\circ}\left(d^{5}-d^{6} \mathrm{MX}_{2}\right)$}

(1) Atom $X$ relaxes along the line $Q-X$.

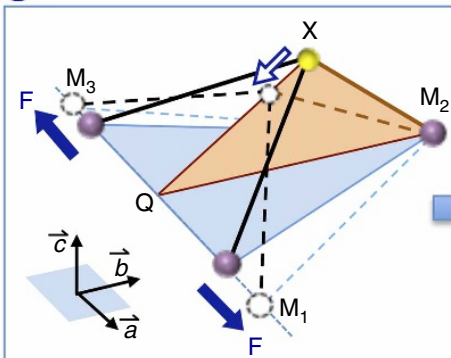

- Atom X relaxes towards point $\mathrm{Q}$.

- $d_{\mathrm{M}_{2} \mathrm{X}}$ increases since $\angle \mathrm{QXM} \mathrm{M}_{2}<90^{\circ}$.

Note: In $d^{0} \mathrm{MX}_{2}$, the $t_{2 g}-p$ coupling is marginal. For the $d^{0} \mathrm{MX}_{2}$ with $\angle \mathrm{QXM} \mathrm{M}_{2}<90^{\circ}$, its deformation mechanism is the same as $d^{1} M X_{2}{ }^{\prime} s$ as shown in (a). For the $d^{0} \mathrm{MX}_{2}$ with $\angle Q X M_{2} \geq 90^{\circ}$, its deformation is same as $d^{5}-d^{6} \mathrm{MX}_{2}$ 's as shown in (b).

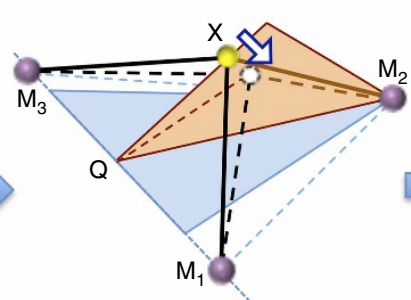

- $\angle \mathrm{XQM}_{2}$ decreases, as a result of the relaxation of the elongated $d_{\mathrm{M}_{2} \mathrm{X}}$

- $\angle \mathrm{M}_{1} \mathrm{XM}_{2}$ and $\angle \mathrm{M}_{3} \mathrm{XM}_{2}$ increase due to the increase in $d_{\mathrm{M}_{1} \mathrm{M}_{2}}$ and $d_{\mathrm{M}_{3} \mathrm{M}_{2}}$
Atom $X$ rotates around the $M_{1}-M_{3}$ axis.

-

(3) Atom $M_{2}$ relaxes along the line $Q-M_{2}$.

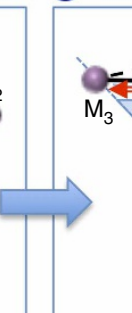

Positive Poisson's ratio

- The $t_{2 g}-p$ coupling in $d^{5}-d^{6} \mathrm{MX}$ aligns along the $\mathrm{M}-\mathrm{X}$ bond and induces no strain energy stored in the $\angle X Q M_{2}$.

- Atoms $\mathrm{M}_{2}$ and $\mathrm{X}$ relax in the $b$ decreasing direction, reducing $\angle \mathrm{M}_{1} \mathrm{XM}_{2}$ and $\angle \mathrm{M}_{3} \mathrm{XM}_{2}$ and releasing the strain energy stored in the increased $\angle M_{1} X M_{2}$ and $\angle M_{3} X M_{2}$

Figure 4 | Deformation mechanism. The solid and dashed $M-X$ bonds indicate, respectively, the initial and final configurations at each relaxation step. The force is applied along the lattice- $a$ direction. The red dashed arrows indicate the direction of the $t_{2 g}-p$ orbital interaction. The hollow blue arrows show the resulting movement of the $X$ and $M_{2}$ atoms within the $Q-X-M_{2}$ plane.

robust in most of the $d^{2}-d^{3} \mathrm{MX}_{2}$ compounds. The less negative Poisson's ratio predicted by HSE06 (Supplementary Table 1) further indicates that the auxetic behaviour originates from the strong $p-d$ coupling. In general, compared with the semilocal SCAN functional, HSE06 yields more localized metal $d$ and chalcogen $p$ orbitals and hence the weaker hybridization between them, which leads to less negative Poisson's ratios in HSE06.

Our predicted in-plane auxetic behaviour is intrinsic in the $1 \mathrm{~T}$ structure without any external engineering and occurs in the elastic region. This is different from the extrinsic auxetic behaviour reported in the epitaxial oxide thin-film ${ }^{53,54}$ and the engineered 2D materials, such as the wrinkled graphene ${ }^{55}$, graphane $^{56}$ and borophane ${ }^{57}$. Recently, the negative Poisson's ratio was also reported in metal nanoplates ${ }^{58}$, pristine graphene $^{59}$ and semi-fluorinated graphene ${ }^{60}$. The negative Poisson's ratio claimed there corresponds to the ratio calculated from $v_{a b}=-\partial \varepsilon_{a} / \partial \varepsilon_{b}$ under large stains, differing from that calculated from $v_{a b}=-\varepsilon_{a} / \varepsilon_{b}$ (the original definition of Poisson's ratio) as we followed here. The Poisson's ratios calculated from $v_{a b}=-\varepsilon_{a} / \varepsilon_{b}$ for pristine graphene ${ }^{59}$ and semi-fluorinated graphene $^{60}$ are actually both positive under a strain $<\sim 15 \%$, and for metal nanoplates, it is also positive under a strain $<\sim 4 \%$.

Finally, it is noteworthy that the auxetic behaviour of $d^{2}-d^{3}$ $\mathrm{MX}_{2}$ compounds is predicted in the high-symmetry $1 \mathrm{~T}$ phase. This phase is known to be metastable or dynamically unstable 
in both $d^{2}$ and $d^{3} \quad \mathrm{MX}_{2}$ compounds ${ }^{44,61-63}$. However, experimentally, relevant phase diagrams of monolayer materials differ from those of bulk materials. The kinetic barriers between the different phases of monolayers may arise and be affected by many external factors, such as interfaces, underlying substrate, temperature, strain and impurities. Therefore, it is not uncommon to observe the undistorted $1 \mathrm{~T}$ phase synthesized experimentally. For instance, although no kinetic barrier is found from first-principles calculations between the unstable 1T phase and dynamically stable distorted $1 \mathrm{~T}$ phase, the undistorted $1 \mathrm{~T}$ monolayer structures of $\mathrm{MoS}_{2}, \mathrm{MoSe}_{2}, \mathrm{WS}_{2}$ and $\mathrm{WSe}_{2}$ are observed from the exfoliation using Li-intercalation method ${ }^{45,64}$. For $\mathrm{MoS}_{2}$, the coexistence of $1 \mathrm{~T}$ and $1 \mathrm{H}$ domains is also observed in the same monolayer ${ }^{46,47}$. Such heterogeneous monolayers with auxetic and non-auxetic domains are particularly intriguing since they could lead to novel functionality.

\section{Methods}

All calculations were performed using density functional theory and the planewave projector augmented-wave ${ }^{65}$ method as implemented in the VASP code $^{66}$. The new SCAN meta-generalized gradient approximation was used ${ }^{67,68}$. SCAN is almost as computationally efficient as PBE-GGA functional, yet it often matches or exceeds the accuracy of the more computationally expensive hybrid functionals in predicting the geometries and energies of diversely bonded systems ${ }^{68}$. Supplementary Table 2 shows our calculated lattice constants for $1 \mathrm{~T}-\mathrm{MX}_{2}$ compounds. They agree very well with available experimental data ${ }^{44}$, especially for groups 4-7 1T- $\mathrm{MX}_{2}$ whose errors are within $1 \%$. An energy cutoff of $500 \mathrm{eV}$ was used. The monolayer structure is modelled in an orthorhombic supercell that contains two formula units (Fig. 1a) and a $20 \AA$ vacuum space inserted in the out-of-plane direction. A $24 \times 14 \times 1 \mathrm{k}$-point grid was used to sample the Brillouin zone during structure relaxation. All atoms were fully relaxed until their atomic forces were $<0.005 \mathrm{eV}^{-1}$. The effects of spin-orbit coupling on the structural deformation are considered to be minor and hence not included in our study.

The Poisson's ratio is calculated from the engineering strain $(\varepsilon)$, which is defined as the change in length $\Delta L$ per unit of the original length $L$, that is, $\varepsilon=\Delta L / L$. The applied uniaxial strain is realized in our calculations by fixing the lattice parameter to a value different from its equilibrium value during structural relaxation. The resulting strain in the transverse direction is extracted from the fully relaxed structure subjected to an applied strain.

Data availability. The authors declare that the data supporting the findings of this study are available within the paper and its Supplementary Information files.

\section{References}

1. Evans, K., Nkansah, M. \& Hutchinson, I. Molecular network design. Nature 353, 124 (1991)

2. Choi, J. \& Lakes, R. Non-linear properties of metallic cellular materials with a negative Poisson's ratio. J. Mater. Sci. 27, 5375-5381 (1992).

3. Lakes, R. \& Elms, K. Indentability of conventional and negative Poisson's ratio foams. J. Compos. Mater. 27, 1193-1202 (1993).

4. Choi, J. \& Lakes, R. Fracture toughness of re-entrant foam materials with a negative Poisson's ratio: experiment and analysis. Int. J. Fract. 80, 73-83 (1996).

5. Evans, K. E. \& Alderson, A. Auxetic materials: functional materials and structures from lateral thinking! Adv. Mater. 12, 617-628 (2000).

6. Greaves, G. N., Greer, A., Lakes, R. \& Rouxel, T. Poisson's ratio and modern materials. Nat. Mater. 10, 823-837 (2011).

7. Lakes, R. Foam structures with a negative Poisson's ratio. Science 235, 1038-1040 (1987).

8. Scarpa, F. Auxetic materials for bioprostheses. IEEE Signal Process. Mag. 25, 125-126 (2008).

9. Avellaneda, M. \& Swart, P. J. Calculating the performance of 1-3 piezoelectric composites for hydrophone applications: an effective medium approach. J. Acoustical Soc. Am. 103, 1449-1467 (1998).

10. Choi, J. \& Lakes, R. Design of a fastener based on negative Poisson's ratio foam. Cell. Polym. 10, 205-212 (1991).

11. Sanami, M., Ravirala, N., Alderson, K. \& Alderson, A. Auxetic materials for sports applications. Proc. Eng. 72, 453-458 (2014).

12. Huang, C. \& Chen, L. Negative Poisson's ratio in modern functional materials. Adv. Mater. 28, 8079-8096 (2016).

13. Lethbridge, Z. A., Walton, R. I., Marmier, A. S., Smith, C. W. \& Evans, K. E. Elastic anisotropy and extreme Poisson's ratios in single crystals. Acta Mater. 58, 6444-6451 (2010).
14. Milstein, F. \& Huang, K. Existence of a negative Poisson ratio in fcc crystals. Phys. Rev. B 19, 2030 (1979).

15. Baughman, R. H., Shacklette, J. M., Zakhidov, A. A. \& Stafström, S. Negative Poisson's ratios as a common feature of cubic metals. Nature 392, 362-365 (1998).

16. Yeganeh-Haeri, A., Weidner, D. J. \& Parise, J. B. Elasticity of a-cristobalite: a silicon dioxide with a negative Poisson's kratio. Science 257, 650-652 (1992).

17. Ogi, H., Fukunaga, M., Hirao, M. \& Ledbetter, H. Elastic constants, internal friction, and piezoelectric coefficient of $\alpha-\mathrm{TeO}_{2}$. Phys. Rev. B 69, 024104 (2004).

18. Williams, J. J., Smith, C. W., Evans, K. E., Lethbridge, Z. A. \& Walton, R. I. Off-axis elastic properties and the effect of extraframework species on structural flexibility of the NAT-type zeolites: simulations of structure and elastic properties. Chem. Mater. 19, 2423-2434 (2007).

19. Gibson, L. J. \& Ashby, M. F. The mechanics of three-dimensional cellular materials. Proc. R. Soc. Lond. A 382, 43-59 (1982).

20. Caddock, B. \& Evans, K. Microporous materials with negative Poisson's ratios. I. Microstructure and mechanical properties. J. Phys. D Appl. Phys. 22, 1877 (1989).

21. He, C., Liu, P. \& Griffin, A. C. Toward negative Poisson ratio polymers through molecular design. Macromolecules 31, 3145 (1998).

22. Milton, G. W. Composite materials with Poisson's ratios close to -1. J. Mech. Phys. Solids 40, 1105-1137 (1992).

23. Miki, M. \& Murotsu, Y. The peculiar behavior of the Poisson's ratio of laminated fibrous composites. JSME Int. J. Ser. 1 Solid Mech. Strength Mater. 32, 67-72 (1989).

24. Song, F., Zhou, J., Xu, X., Xu, Y. \& Bai, Y. Effect of a negative Poisson ratio in the tension of ceramics. Phys. Rev. Lett. 100, 245502 (2008).

25. Wojciechowski, K. Constant thermodynamic tension Monte Carlo studies of elastic properties of a two-dimensional system of hard cyclic hexamers. Mol. Phys. 61, 1247-1258 (1987).

26. Ortiz, A. U., Boutin, A., Fuchs, A. H. \& Coudert, F.-X. Anisotropic elastic properties of flexible metal-organic frameworks: how soft are soft porous crystals? Phys. Rev. Lett. 109, 195502 (2012).

27. Babaee, S. et al. 3D Soft metamaterials with negative Poisson's ratio. Adv. Mater. 25, 5044-5049 (2013).

28. Schenk, M. \& Guest, S. D. Geometry of Miura-folded metamaterials. Proc. Natl Acad. Sci. 110, 3276-3281 (2013).

29. Wei, Z. Y., Guo, Z. V., Dudte, L., Liang, H. Y. \& Mahadevan, L. Geometric mechanics of periodic pleated origami. Phys. Rev. Lett. 110, 215501 (2013).

30. Silverberg, J. L. et al. Using origami design principles to fold reprogrammable mechanical metamaterials. Science 345, 647-650 (2014).

31. Rothenburg, L., Berlin, A. A. \& Bathurst, R. J. Microstructure of isotropic materials with negative Poisson's ratio. Nature 354, 470-472 (1991).

32. Baughman, R. H. \& Galvão, D. S. Crystalline networks with unusual predicted mechanical and thermal properties. Nature 365, 735-737 (1993).

33. Lakes, R. Deformation mechanisms in negative Poisson's ratio materials: structural aspects. J. Mater. Sci. 26, 2287-2292 (1991).

34. Jiang, J.-W. \& Park, H. S. Negative Poisson's ratio in single-layer black phosphorus. Nat. Commun. 5, 4727 (2014).

35. Du, Y. et al. Auxetic black phosphorus: a 2D material with negative Poisson's ratio. Nano Lett. 16, 6701-6708 (2016).

36. Gomes, L. C., Carvalho, A. \& Castro Neto, A. H. Enhanced piezoelectricity and modified dielectric screening of two-dimensional group-IV monochalcogenides. Phys. Rev. B 92, 214103 (2015).

37. Han, J. et al. Negative Poisson's ratios in few-layer orthorhombic arsenic: first-principles calculations. Appl. Phys. Express 8, 041801 (2015).

38. Mannix, A. J. et al. Synthesis of borophenes: anisotropic, two-dimensional boron polymorphs. Science 350, 1513-1516 (2015).

39. Zhang, S. et al. Penta-graphene: a new carbon allotrope. Proc. Natl Acad. Sci. 112, 2372-2377 (2015)

40. Yagmurcukardes, M. et al. Pentagonal monolayer crystals of carbon, boron nitride, and silver azide. J. Appl. Phys. 118, 104303 (2015).

41. Özçelik, V. O., Cahangirov, S. \& Ciraci, S. Stable single-layer honeycomblike structure of silica. Phys. Rev. Lett. 112, 246803 (2014).

42. Wang, Y., Li, F., Li, Y. \& Chen, Z. Semi-metallic $\mathrm{Be}_{5} \mathrm{C}_{2}$ monolayer global minimum with quasi-planar pentacoordinate carbons and negative Poisson's ratio. Nat. Commun. 7, 11488 (2016).

43. Wang, Q. H., Kalantar-Zadeh, K., Kis, A., Coleman, J. N. \& Strano, M. S Electronics and optoelectronics of two-dimensional transition metal dichalcogenides. Nat. Nanotechnol. 7, 699-712 (2012).

44. Wilson, J. \& Yoffe, A. The transition metal dichalcogenides discussion and interpretation of the observed optical, electrical and structural properties. $A d v$. Phys. 18, 193-335 (1969).

45. Py, M. \& Haering, R. Structural destabilization induced by lithium intercalation in $\mathrm{MoS}_{2}$ and related compounds. Can. J. Phys. 61, 76-84 (1983).

46. Eda, G. et al. Coherent atomic and electronic heterostructures of single-layer $\mathrm{MoS}_{2}$. ACS Nano 6, 7311-7317 (2012). 
47. Lin, Y.-C., Dumcenco, D. O., Huang, Y.-S. \& Suenaga, K. Atomic mechanism of the semiconducting-to-metallic phase transition in single-layered $\mathrm{MoS}_{2}$. Nat. Nanotechnol. 9, 391-396 (2014).

48. Chhowalla, M. et al. The chemistry of two-dimensional layered transition metal dichalcogenide nanosheets. Nat. Chem. 5, 263-275 (2013).

49. Yu, L., Ruzsinszky, A. \& Perdew, J. P. Bending two-dimensional materials to control charge localization and Fermi-level shift. Nano Lett. 16, 2444-2449 (2016).

50. Stott, P. J., Mitchell, R., Alderson, K. \& Alderson, A. A growth industry. Mater. World 8, 12-14 (2000).

51. Heyd, J., Scuseria, G. E. \& Ernzerhof, M. Hybrid functionals based on a screened Coulomb potential. J. Chem. Phys. 118, 8207-8215 (2003).

52. Ruzsinszky, A., Perdew, J. P., Csonka, G. I., Scuseria, G. E. \& Vydrov, O. A. Understanding and correcting the self-interaction error in the electrical response of hydrogen chains. Phys. Rev. A 77, 060502 (2008).

53. Sandiumenge, F. et al. Competing misfit relaxation mechanisms in epitaxial correlated oxides. Phys. Rev. Lett. 110, 107206 (2013).

54. Aguirre-Tostado, F. et al. Elastic anomaly for $\mathrm{Sr}^{\mathrm{TiO}} 3$ thin films grown on $\mathrm{Si}$ (001). Phys. Rev. B 70, 201403 (2004).

55. Grima, J. N. et al. Tailoring graphene to achieve negative Poisson's ratio properties. Adv. Mater. 27, 1455-1459 (2015).

56. Cadelano, E., Palla, P. L., Giordano, S. \& Colombo, L. Elastic properties of hydrogenated graphene. Phys. Rev. B 82, 235414 (2010).

57. Kou, L. et al. Auxetic and ferroelastic borophane: a novel 2D material with negative Possion's ratio and switchable dirac transport channels. Nano Lett. 16, 7910-7914 (2016).

58. Ho, D. T., Park, S.-D., Kwon, S.-Y., Park, K. \& Kim, S. Y. Negative Poisson's ratios in metal nanoplates. Nat. Commun. 5, 3255 (2014).

59. Jiang, J.-W., Chang, T., Guo, X. \& Park, H. S. Intrinsic negative Poisson's ratio for single-layer graphene. Nano Lett. 16, 5286-5290 (2016).

60. Qin, R., Zheng, J. \& Zhu, W. Sign-tunable Poisson's ratio in semi-fluorinated graphene. Nanoscale 9, 128-133 (2017).

61. Duerloo, K.-A. N., Li, Y. \& Reed, E. J. Structural phase transitions in two-dimensional Mo-and W-dichalcogenide monolayers. Nat. Commun. 5, 4214 (2014).

62. Tongay, S. et al. Monolayer behaviour in bulk $\operatorname{ReS}_{2}$ due to electronic and vibrational decoupling. Nat. Commun. 5, 3252 (2014).

63. Fang, C., Wiegers, G., Haas, C. \& De Groot, R. Electronic structures of, and in the real and the hypothetical undistorted structures. J. Phys. Condens. Matter 9, 4411 (1997).

64. Ambrosi, A., Sofer, Z. \& Pumera, M. $2 \mathrm{H} \rightarrow 1 \mathrm{~T}$ phase transition and hydrogen evolution activity of $\mathrm{MoS}_{2}, \mathrm{MoSe}_{2}, \mathrm{WS}_{2}$ and $\mathrm{WSe}_{2}$ strongly depends on the MX 2 composition. Chem. Commun. 51, 8450-8453 (2015).

65. Blöchl, P. E. Projector augmented-wave method. Phys. Rev. B 50, 17953 (1994).

66. Kresse, G. \& Furthmüller, J. Efficiency of ab-initio total energy calculations for metals and semiconductors using a plane-wave basis set. Comput. Mater. Sci. 6, 15-50 (1996).

67. Sun, J., Ruzsinszky, A. \& Perdew, J. P. Strongly constrained and appropriately normed semilocal density functional. Phys. Rev. Lett. 115, 036402 (2015).
68. Sun, J. et al. Accurate first-principles structures and energies of diversely bonded systems from an efficient density functional. Nat. Chem. 8, 831-836 (2016).

\section{Acknowledgements}

We thank John P. Perdew for valuable scientific discussions and comments on the manuscript. We also thank Richard C. Remsing and Jefferson E. Bates for the comments on the manuscript. This research was supported as part of the Center for the Computational Design of Functional Layered Materials (CCDM), an Energy Frontier Research Center funded by the U.S. Department of Energy (DOE), Office of Science, Basic Energy Sciences (BES), under Award \#DE-SC0012575. This research used resources of the National Energy Research Scientific Computing Center, a DOE Office of Science User Facility supported by the Office of Science of the U.S. Department of Energy under Contract No. DE-AC02-05CH11231. This research was also supported in part by the National Science Foundation through major research instrumentation grant number CNS-09-58854.

\section{Author contributions}

L.Y. designed the project, performed the calculations and wrote the manuscript. Q.Y. and A.R. contributed to analysing the results and writing the manuscript.

\section{Additional information}

Supplementary Information accompanies this paper at http://www.nature.com/ naturecommunications

Competing interests: The authors declare no competing financial interests.

Reprints and permission information is available online at http://npg.nature.com/ reprintsandpermissions/

How to cite this article: Yu, L. et al. Negative Poisson's ratio in 1T-type crystalline two-dimensional transition metal dichalcogenides. Nat. Commun. 8, 15224 doi: 10.1038/ncomms15224 (2017).

Publisher's note: Springer Nature remains neutral with regard to jurisdictional claims in published maps and institutional affiliations.

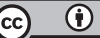

This work is licensed under a Creative Commons Attribution 4.0 International License. The images or other third party material in this article are included in the article's Creative Commons license, unless indicated otherwise in the credit line; if the material is not included under the Creative Commons license, users will need to obtain permission from the license holder to reproduce the material To view a copy of this license, visit http://creativecommons.org/licenses/by/4.0/

(C) The Author(s) 2017 